\title{
Initial clinical experience treating patients with palliative radiotherapy for malignant pleural mesothelioma on the Halcyon ${ }^{\mathrm{TM}}$ linear accelerator
}

\author{
Andrew R. Barsky^, Michele M. Kim, Russell Maxwell, Amberly Mendes, Christopher M. Wright, \\ Emily J. Anstadt, Sally McNulty, Lei Dong, James M. Metz, Steven J. Feigenberg, Taoran Li", \\ Keith A. Cengel ${ }^{\#}$
}

Department of Radiation Oncology, Perelman Center for Advanced Medicine, Perelman School of Medicine of the University of Pennsylvania, Philadelphia, PA, USA

Contributions: (I) Conception and design: AR Barsky, MM Kim, T Li, KA Cengel; (II) Administrative support: L Dong, JM Metz, SJ Feigenberg, T Li, KA Cengel; (III) Provision of study materials or patients: S McNulty, T Li, KA Cengel; (IV) Collection and assembly of data: AR Barsky, MM Kim, A Mendes, T Li, KA Cengel; (V) Data analysis and interpretation: AR Barsky, MM Kim, T Li, KA Cengel; (VI) Manuscript writing: All authors; (VII) Final approval of manuscript: All authors.

\#These authors shared co-senior authorship.

Correspondence to: Andrew R. Barsky, MD. Department of Radiation Oncology, Perelman Center for Advanced Medicine, TRC R West, 3400 Civic Center Boulevard, Philadelphia, PA 19104, USA. Email: Andrew.Barsky@uphs.upenn.edu.

Background: Radiation therapy (RT) can provide effective symptomatic palliation in patients with malignant pleural mesothelioma (MPM). Advances in RT technology, including intensity-modulated RT (IMRT) and volumetric-modulated arc therapy (VMAT), have improved treatment conformality, potentially improving the therapeutic ratio of RT. A novel 6-MV flattening-filter-free O-ring linear accelerator, Halcyon $^{\mathrm{TM}}$ (Varian Medical Systems, Palo Alto, CA, USA), was built to provide such advanced therapies, while possibly reducing treatment time. Here, we report the initial clinical experience using Halcyon ${ }^{\mathrm{TM}}$ to deliver palliative RT for patients with MPM.

Methods: We retrospectively assessed consecutive patients with MPM who received thoracic RT on Halcyon $^{\mathrm{TM}}$. Their electronic medical records were reviewed for clinical, RT planning, treatment timing, and image-guidance RT (IGRT) data.

Results: Four patients with metastatic MPM received palliative RT on Halcyon ${ }^{\text {TM }}$ between 1/2017-1/2020 for severe pain (50\%), dysphagia (25\%), or dyspnea (25\%). Targets included a combination of pleura, chest wall, lung, hilum, and mediastinum, with patient-specific dose and fractionation regimens ranging from 20-45 Gy in 5-15 fractions, and 75\% of patients receiving concurrent systemic therapy. Pre-specified target and organ-at-risk constraints were met for nearly all plans. At a median follow-up of 2.2 months (range, 1.6-7.1 months), all patients experienced either improved (75\%) or stable (25\%) tumor-related symptoms following palliative RT. The mean $3 \mathrm{D}$ vector couch correction was $0.67 \pm 0.15 \mathrm{~cm}$. The mean beam-on, treatment (beam-on plus cone-beam computed tomography times), and approximated total room usage times were $1.6 \pm 0.2,1.8 \pm 0.2$, and $9.8 \pm 0.2 \mathrm{~min}$, respectively. Grade 2 fatigue and cough occurred in $25 \%$ and $25 \%$ of patients, and no patients experienced Grade $\geq 3$ toxicity.

Conclusions: In this initial clinical experience treating patients with palliative RT for MPM on $\operatorname{Halcyon}^{\mathrm{TM}}$, treatment provided symptom palliation and local control across multiple palliative scenarios, with minimal toxicity, acceptable dosimetry, and setup corrections and treatment times that compared favorably with other published experiences of MPM RT. Palliative RT on Halcyon ${ }^{\text {TM }}$ can provide patients with MPM quick and safe tumor-related symptom relief, even in a frail, elderly population.

^ ORCID: 0000-0002-9761-3000. 
Keywords: Mesothelioma; metastases; radiotherapy (RT)

Submitted Feb 17, 2020. Accepted for publication Jul 14, 2020.

doi: 10.21037/apm-20-385

View this article at: http://dx.doi.org/10.21037/apm-20-385

\section{Introduction}

Malignant pleural mesothelioma (MPM) is an aggressive cancer of the mesothelial cells of the pleura, affecting roughly one in 100,000 people annually in America (1). MPM management is derived from the patient's cancer stage, tumor histology, medical co-morbidities, and functional status. The National Comprehensive Cancer Network (NCCN) guidelines recommend that medically fit patients with clinical stage I-IIIA disease and epithelioid or biphasic histology undergo surgery (if feasible) and chemotherapy, with consideration of adjuvant radiotherapy (RT). For patients with clinical stage IIIB-IV disease, sarcomatoid histology, or who are medically inoperable, chemotherapy, observation, or best supportive care are the recommended options. The guidelines state that RT provides effective palliation of chest pain, bronchial or esophageal obstruction, or other MPM-related symptomatic sites (2). Additionally, the expert opinion regarding the use of RT in MPM from the National Cancer Institute Thoracic Malignancy Steering Committee, International Association for the Study of Lung Cancer, and Mesothelioma Applied Research Foundation considers pre- or post-operative RT, procedure tract RT, or focal MPM symptom palliative RT all to be valid indications for RT. Thus, RT may play a role in curative and palliative management of MPM(3).

The safe delivery of RT can be challenging using older techniques, such as two-dimensional and three-dimensional conformal RT (3D-CRT), as large volumes of lung and other critical organs may receive high doses, leaving the potential for severe radiation pneumonitis, among other toxicities $(4,5)$. Consequently, intensity-modulated RT (IMRT), a more advanced radiation delivery technique, has been used to improve treatment conformality, reduce organ-at-risk (OAR) doses, and possibly increase the therapeutic ratio of treatment (6). Volumetric-modulated arc therapy (VMAT), is a form of IMRT in which RT is delivered in continuous, dynamic arcs. While published reports of the use of VMAT in MPM are limited, it has been shown that VMAT can significantly improve MPM treatment homogeneity and conformity indices and shorten treatment delivery times as compared to fixed-field $\operatorname{IMRT}(7)$, with reasonable toxicity (8).

Recently, a 6-MV flattening-filter-free (FFF) O-ring linear accelerator (linac), Halcyon ${ }^{\mathrm{TM}}$ (Varian Medical Systems, Palo Alto, CA, USA), was built to improve treatment speed and throughput relative to a $\mathrm{C}$-arm linac (CAL) (9). RT with FFF beams can increase dose rate, and decrease head scatter and penumbra relative to RT with flattening-filtered beams (10). Pre-clinical treatment planning studies comparing RT on Halcyon ${ }^{\mathrm{TM}}$ to RT on CALs in head-and-neck cancer $(9,11,12)$, pediatric cancers (13), prostate cancer (12), breast cancer $(12,14,15)$, and stereotactic RT $(11,16)$ showed overall similar plan quality and faster calculated treatment times with Halcyon $^{\text {TM }}$. Aside from reports of the use of Halcyon ${ }^{\mathrm{TM}}$ to treat patients with breast cancer and gynecologic cancers showing fast beam-on, treatment, and in-room times for patients, with comparable OAR doses and toxicity to breast and pelvic RT on CALs $(17,18)$, reports of the clinical use of Halcyon ${ }^{\mathrm{TM}}$ are limited.

Herein, we report our initial clinical experience treating patients for MPM with palliative RT on Halcyon ${ }^{\mathrm{TM}}$, which represents the first published experience of the use of Halcyon $^{\text {TM }}$ to treat MPM and one of the only published experiences of the clinical use of Halcyon ${ }^{\mathrm{TM}}$ to date. We estimated that MPM RT on Halcyon ${ }^{\text {TM }}$ would provide comparable acute toxicity and dosimetry to MPM RT on a CAL, with shorter treatment times. We present the following article in accordance with the STROBE reporting checklist (available at http://dx.doi.org/10.21037/apm-20-385).

\section{Methods}

With institutional review board approval, we conducted a retrospective case series analysis of patients who received thoracic RT for MPM on Halcyon ${ }^{\text {TM }}$ at our institution between $1 / 2017$ and $1 / 2020$. The only exclusion criterion was receipt of at least one fraction of RT on a nonHalcyon $^{\mathrm{TM}}$ linac. Patients' electronic medical records were reviewed through 6/2020 for clinical, RT planning, treatment timing, and image-guidance RT (IGRT) data. This 
study was conducted in accordance with the Declaration of Helsinki (as revised in 2013). The study was approved by the institutional review board of the Hospital of the University of Pennsylvania (Protocol No. 831888) and individual consent for this retrospective analysis was waived.

\section{Prescriptions and constraints}

Computed tomography (CT) simulation was performed in the supine position, using a knee-foot lock, arm shuttle, and four-dimensional (4D)-CT, compression belt, and Vac-Lok ${ }^{\mathrm{TM}}$ bag (CIVCO Radiotherapy, Orange City, Iowa, USA) when clinically indicated. Symptomatic sites were marked on the skin with wire at the discretion of the treating physician. Contouring consisted of delineation of symptomatic gross disease as the gross tumor volume (GTV), adjusted to an internal target volume (ITV) if 4DCT was used, to account for tumor motion during patient respiration. The GTV or ITV was expanded uniformly by $0.5-0.7 \mathrm{~cm}$ to create a planning target volume (PTV). Patients received RT to gross, symptomatic, thoracic disease in 3-4 Gy fractions to total doses ranging from 20-45 Gy at the discretion of the treating physician, with attention to the patients' overall prognoses, location of disease, and logistical considerations.

RT planning was completed in Eclipse $\mathrm{E}^{\mathrm{TM}}$ (Varian Medical Systems, Palo Alto, CA, USA) version 15.6 with 6-MV FFF photon VMAT. Dosimetric target coverage and OAR constraints were patient-specific given the variety of RT doses and fractionations used and are listed by patient in Table 1.

\section{Treatment planning and image guidance}

RT plans were created in Eclipse ${ }^{\mathrm{TM}}$ using one isocenter with two arcs for all patients. Characteristic VMAT planning techniques with rotated collimators were utilized. Beammodulation was accomplished using $1.0-\mathrm{cm}$ dual-layer stacked and staggered multi-leaf collimators. Representative images of each patient's RT plan are shown in Figure 1.

\section{Data analysis}

The primary objective was to describe the initial clinical experience treating patients with MPM with palliative RT on Halcyon ${ }^{\mathrm{TM}}$. The specific clinical endpoints assessed were the variety of patients and treatment techniques used, symptomatic palliation, treatment toxicity, dosimetric parameters, couch corrections, and treatment timing. Patient-reported symptomatic palliation was assessed clinically at follow-up patient encounters. Acute toxicity data was assessed with the common terminology criteria for adverse events (CTCAE) version 5.0 grading system at scheduled office visits during and following RT. RT dosimetry was assessed using dose-volume histogram (DVH) data for PTV coverage and OAR sparing. Couch corrections were assessed by computing the mean couch correction for all RT fractions from daily cone-beam CT (CBCT) to bony anatomy from the time of simulation. Treatment timing was assessed using beam-on time delivered to an electronic portal imaging device (EPID) for each patient's specific plan, mean treatment time [time for a CBCT delivered to the kilovoltage $(\mathrm{kV})$ imaging portal plus beam-on time], and an approximated mean total room usage time, because such times were not routinely recorded for MPM patients on Halcyon $^{\mathrm{TM}}$. Mean total room usage time was approximated based upon data from a previously published experience of Halcyon ${ }^{\mathrm{TM}} \mathrm{RT}$ to treat breast cancer, such that mean treatment time (CBCT plus beam-on time), $4.4 \mathrm{~min}$, from that study was subtracted from the mean total room usage time from that study, $12.4 \mathrm{~min}$, to obtain a benchmark value for mean room usage time minus mean treatment time, which was $8.0 \mathrm{~min}(17)$. As such, mean total room usage time in this analysis was approximated by adding $8.0 \mathrm{~min}$ to the mean treatment time.

\section{Statistics}

Descriptive statistics (medians, means, ranges, standard deviations when relevant for continuous variables, and percentages for categorical variables) were used to describe data. Couch correction and treatment time data were qualitatively compared to published reference values. Data analysis was performed with MATLAB R2018a Statistics Toolbox software package (The MathWorks Inc., Natick, MA, USA).

\section{Results}

\section{Patient clinico-pathologic and treatment details}

Four consecutive patients met inclusion criteria for analysis. The median age at RT in our cohort was 81 years (range, $75-83$ years). Patients had a median Eastern Cooperative Oncology Group (ECOG) performance status of two (range, one-two), and a median follow-up interval of 2.2 months (range, 1.6-7.1 months). 
Table 1 Dosimetric parameters of targets and OARs for each patient course

\begin{tabular}{|c|c|}
\hline Variables & Value (\%) \\
\hline \multicolumn{2}{|c|}{ Patient 1 (20 Gy, 5 fractions) } \\
\hline \multicolumn{2}{|c|}{ PTV (D95\% >90\%) } \\
\hline D95\% & 90.6 \\
\hline V110\% & 0 \\
\hline \multicolumn{2}{|c|}{ Lungs-ITV (mean <5.5 Gy) } \\
\hline Mean (Gy) & 4.6 \\
\hline \multicolumn{2}{|c|}{ Esophagus (maximum <16 Gy) } \\
\hline Maximum (Gy) & 16.2 \\
\hline \multicolumn{2}{|c|}{ Spinal cord (plan sum maximum <50 Gy) } \\
\hline Maximum (Gy) & 49.6 \\
\hline \multicolumn{2}{|c|}{ Patient 2 (30 Gy, 10 fractions) } \\
\hline \multicolumn{2}{|c|}{ PTV (D95\% $\geq 92.5 \%)$} \\
\hline D95 & 95.1 \\
\hline V110 & 0 \\
\hline \multicolumn{2}{|c|}{ Lungs-ITV (mean <8 Gy) } \\
\hline Mean (Gy) & 5.3 \\
\hline \multicolumn{2}{|c|}{ Esophagus (maximum <40 Gy) } \\
\hline Maximum (Gy) & 30.5 \\
\hline \multicolumn{2}{|c|}{ Heart (mean <11.5 Gy) } \\
\hline Mean (Gy) & 8.5 \\
\hline \multicolumn{2}{|c|}{ Spinal cord (maximum <16 Gy) } \\
\hline Maximum (Gy) & 11.8 \\
\hline \multicolumn{2}{|c|}{ Patient 3 (32 Gy, 8 fractions) } \\
\hline \multicolumn{2}{|c|}{ PTV (D95\% >90\%) } \\
\hline D95 & 96.0 \\
\hline V110 & 0 \\
\hline \multicolumn{2}{|c|}{ Lungs-GTV (mean <8 Gy) } \\
\hline Mean (Gy) & 0.1 \\
\hline \multicolumn{2}{|c|}{ Esophagus (maximum <5 Gy) } \\
\hline Maximum (Gy) & 0.8 \\
\hline \multicolumn{2}{|c|}{ Heart (maximum < 5 Gy) } \\
\hline Maximum (Gy) & 4.2 \\
\hline \multicolumn{2}{|c|}{ Spinal cord (maximum <10 Gy) } \\
\hline Maximum (Gy) & 5.0 \\
\hline
\end{tabular}

Table 1 (continued)
Table 1 (continued)

\begin{tabular}{lc}
\hline Variables & Value (\%) \\
\hline Patient 4 (45 Gy, 15 fractions) & 94.3 \\
PTV (D95\% $\geq 92 \%)$ & 0 \\
D95 & \\
V110 & 7.7 \\
Lungs-ITV (mean <10 Gy) & \\
Mean (Gy) & 11.9 \\
Esophagus (mean <12 Gy) & \\
Mean (Gy) & 3.5 \\
Heart (maximum <16 Gy) & \\
Maximum (Gy) & 34.9 \\
Spinal cord (maximum <35 Gy) & \\
Maximum (Gy)
\end{tabular}

OAR, organ at risk; Gy, Gray; PTV, planning target volume; D95\%, minimum dose received by $95 \%$ of the PTV; V110, volume receiving at least $110 \%$ of the pre-scription dose; ITV, internal target volume; GTV, gross tumor volume.

All patients $(100 \%, n=4)$ were treated for Stage IV MPM with the goal of palliation of tumor-related dyspnea $(25 \%, \mathrm{n}=1)$, dysphagia $(25 \%, \mathrm{n}=1)$, or narcotic-requiring pain $(50 \%, n=2)$. RT was most-commonly administered for right-sided disease $(75 \%, n=3)$. MPM histologies treated included epithelioid $(50 \%, \mathrm{n}=2)$, biphasic $(25 \%, \mathrm{n}=1)$, and sarcomatoid $(25 \%, \mathrm{n}=1)$.

Patients received systemic therapy concurrently with RT and sequentially in $75 \%(n=3)$ of cases, and sequentiallyonly in $25 \%(n=1)$. Concurrent agents consisted of pemetrexed for one patient $(33 \%)$ and pembrolizumab for two patients $(67 \%)$. Sequential-only therapy was gemcitabine $(100 \%, \mathrm{n}=1)$.

Patients' RT course details are shown in Table 2. All $(100 \%, n=4)$ were treated in supine position with VMAT. Motion management utilizing 4D-CT was performed in three patients $(75 \%)$, with use of a compression belt in 1 of the $3(33 \%)$. All patients $(100 \%, n=4)$ were immobilized using a knee-foot lock and arm shuttle. Additionally, a Vac$\mathrm{Lok}^{\mathrm{TM}}$ bag was used in 2 of the 4 patients $(50 \%)$. Treatment targets, doses, and fractionations for each patient were: (I) pleura, mediastinum, lung, and hilum, 20 Gy in 5 fractions (Figure 1A), (II) pleura and mediastinum, 30 Gy in 10 fractions (Figure 1B), (III) pleura and chest 

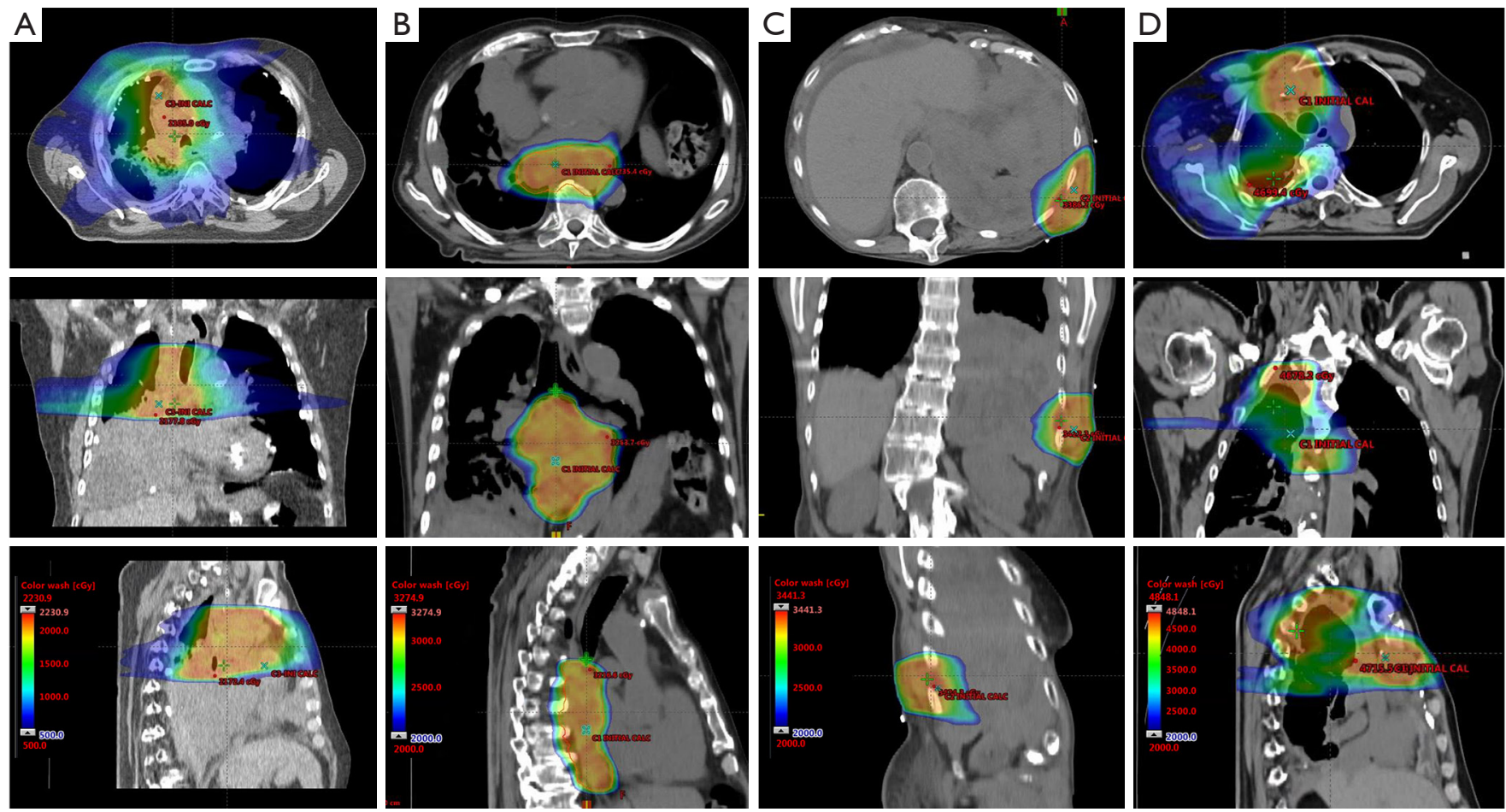

Figure 1 Radiotherapy dose color wash for all patients. Representative axial (top), coronal (middle), and sagittal (bottom) images of radiotherapy dose color wash for all four patients' treatments. Doses delivered were 20 Gy in 5 fractions (A), 30 Gy in 10 fractions (B), 32 Gy in 8 fractions (C), and 45 Gy in 15 fractions (D).

wall, 32 Gy in 8 fractions (Figure 1C), and (IV) pleura, chest wall, mediastinum, and lung, 45 Gy in 15 fractions (Figure 1D). The median PTV size was $285.8 \mathrm{~cm}^{3}$ (range, $\left.75.7-489.4 \mathrm{~cm}^{3}\right)$. All patients $(100 \%, \mathrm{n}=4)$ received daily $\mathrm{kV}$ CBCT for IGRT.

\section{Dosimetry}

Each patient's RT plan's dosimetric parameters for targets and OARs are listed in Table 1. All plans $(100 \%, n=4)$ met the prescribed target coverage constraints. Nearly all plans $(75 \%, n=3)$ met the prescribed OAR constraints for lungsITV or lungs-GTV, esophagus, heart, and spinal cord, when applicable. The one plan that exceeded an OAR constraint delivered 16.2 Gy to the esophagus, with a constraint of 16 Gy maximum esophageal dose. This was exceeded at the discretion of the treating physician in order to maximize target coverage.

\section{Palliation}

At 2.2 months of median follow-up (range, 1.6-7.1 months), each patient experienced either improved $(75 \%, n=3)$ or stable $(25 \%, \mathrm{n}=1)$ tumor-related symptoms following symptom-directed RT on Halcyon ${ }^{\mathrm{TM}}$. Specifically, one patient experienced dyspnea on exertion resulting in hypoxia requiring home oxygen, nebulizers, and steroid treatments, in the setting of tumor compression of the right mainstem bronchus. Within a month of completion of RT, he experienced tumor regression to the point that his bronchial compression was reduced, as demonstrated on CT, and his supplemental oxygen requirement remained stable for the remainder of his clinical course (Figure $2 A$ ). The patient who presented with dysphagia reported multiple weeks of progressive regurgitation and a sense that many foods were unable to be swallowed, prior to RT. These symptoms were corroborated with bulky mediastinal disease compressing the esophagus. Within three weeks of completion of mediastinal RT, an interval CT chest demonstrated stable, but necrotic irradiated disease, and his swallowing function improved to the point that he was able to eat foods of most consistencies (Figure 2B). Both patients who presented with severe chest wall pain requiring narcotics at radiation oncology consultation reported complete resolution of pain 
Table 2 Details of radiotherapy course

\begin{tabular}{|c|c|}
\hline Variables & Value \\
\hline \multicolumn{2}{|l|}{ Laterality, n [\%] } \\
\hline Left & $1[25]$ \\
\hline Right & $3[75]$ \\
\hline \multicolumn{2}{|l|}{ Target, n [\%] } \\
\hline Pleura, chest wall & $1[25]$ \\
\hline Pleura, mediastinum & $1[25]$ \\
\hline Pleura, chest wall, mediastinum, lung & $1[25]$ \\
\hline Pleura, mediastinum, lung, hilum & $1[25]$ \\
\hline \multicolumn{2}{|l|}{ Delivered dose and fractionation, $\mathrm{n}$ [\%] } \\
\hline 20 Gy, 5 fractions & $1[25]$ \\
\hline 30 Gy, 10 fractions & $1[25]$ \\
\hline 32 Gy, 8 fractions & $1[25]$ \\
\hline 45 Gy, 15 fractions & $1[25]$ \\
\hline \multicolumn{2}{|l|}{ PTV size $\left(\mathrm{cm}^{3}\right)$} \\
\hline Median & 285.8 \\
\hline Range & $75.7-489.4$ \\
\hline \multicolumn{2}{|l|}{ Modality, n [\%] } \\
\hline VMAT & $4[100]$ \\
\hline \multicolumn{2}{|l|}{ Motion management, $\mathrm{n}[\%]$} \\
\hline 4D-CT alone & $2[50]$ \\
\hline 4D-CT with compression belt & $1[25]$ \\
\hline None & $1[25]$ \\
\hline \multicolumn{2}{|l|}{ Immobilization, n [\%] } \\
\hline Knee-foot lock, arm shuttle & $2[50]$ \\
\hline Vac-Lok ${ }^{\mathrm{TM}}$ bag, knee-foot lock, arm shuttle & $2[50]$ \\
\hline \multicolumn{2}{|l|}{ IGRT, n [\%] } \\
\hline kV CBCT & $4[100]$ \\
\hline \multicolumn{2}{|l|}{ Systemic therapy, n [\%] } \\
\hline Concurrent-only & $0[0]$ \\
\hline Sequential-only ${ }^{\dagger}$ & $1[25]$ \\
\hline Concurrent $^{\ddagger}$ and sequential & $3[75]$ \\
\hline None & $0[0]$ \\
\hline
\end{tabular}

${ }^{\dagger}$, sequential-only therapy consisted of gemcitabine; ${ }^{\ddagger}$, concurrent agents consisted of pemetrexed for one patient and pembrolizumab for two patients. Gy, Gray; PTV, planning target volume; VMAT, volumetric-modulated arc therapy; 4D-CT, four-dimensional computed tomography; IGRT, image-guided radiotherapy; kV $\mathrm{CBCT}$, kilovoltage cone-beam computed tomography. at the irradiated sites and cessation of narcotic use within 1-2 weeks after RT. All patients expired due to progressive disease outside of the irradiated fields.

\section{Acute toxicity}

No patients $(0 \%, \mathrm{n}=0)$ experienced any Grade 3 or higher acute toxicities. Grade 1 toxicities observed included dyspnea $(100 \%, n=4)$, fatigue $(75 \%, n=3)$, cough $(50 \%$, $\mathrm{n}=2)$, and nausea $(50 \%, \mathrm{n}=2)$. Grade 2 toxicities observed included fatigue $(25 \%, \mathrm{n}=1)$ and cough $(25 \%, \mathrm{n}=1)$.

\section{Patient setup uncertainty and IGRT experience}

The mean $3 \mathrm{D}$ vector couch correction from superficial marker positioning to daily CBCT for all fractions of all patients' setups on Halcyon ${ }^{\mathrm{TM}}$ was $0.67 \pm 0.15 \mathrm{~cm}$ (Figure 3).

\section{Treatment time and throughput analysis}

The mean beam-on time for all patients was $1.6 \pm 0.2 \mathrm{~min}$. The mean treatment time for all patients was $1.8 \pm 0.2 \mathrm{~min}$. The approximated mean total treatment room usage time for all patients was $9.8 \pm 0.2 \mathrm{~min}$ (Table 3).

\section{Discussion}

In this case series, we describe the initial clinical experience delivering RT on Halcyon ${ }^{\mathrm{TM}}$ for MPM, and one of the first published experiences describing the clinical use of Halcyon $^{\mathrm{TM}}$. We demonstrate its use in multiple palliative clinical scenarios, its palliation outcomes, acute toxicity, dosimetric data, couch corrections, and treatment speed.

RT on Halcyon ${ }^{\mathrm{TM}}$ provided symptomatic palliation for all patients $(100 \%, n=4)$, with three patients $(75 \%)$ reporting complete relief of tumor-related symptoms, and one patient (25\%) reporting stable tumor-related symptoms, which remained durable through the remainder of their clinical courses. This was accomplished using a variety of dosing and fractionation schedules, including standard-dose and high-dose regimens. This experience compared favorably with other published experiences of palliative RT for MPM, and adds to the limited body of such literature. In a 189-patient experience of palliative RT for MPM, $50 \%$ of patients receiving RT in 4 Gy fractions to a median dose of $36 \mathrm{~Gy}$, had local responses, with recurrences of pain occurring at a median of 69 days from treatment (19). In a 19 -patient experience of 

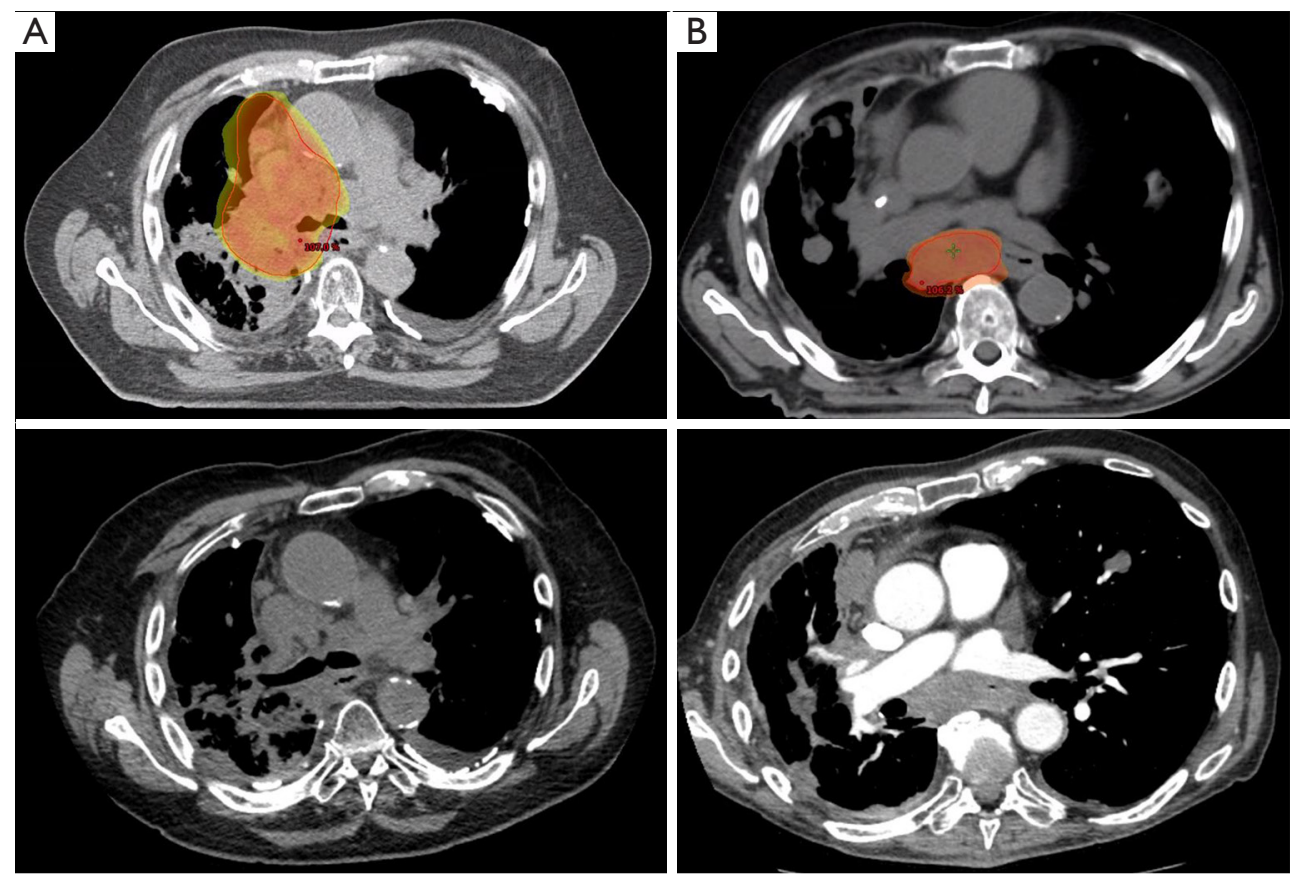

Figure 2 Tumor response following palliative radiotherapy. Representative axial chest computed tomography images of symptomatic radiotherapy targets (top) resulting in severe dyspnea due to right mainstem bronchial compression (A) and dysphagia due to esophageal compression (B). Tumor response (bottom) at 1 month following 20 Gy in 5 fractions (A) and 30 Gy in 10 fractions (B).

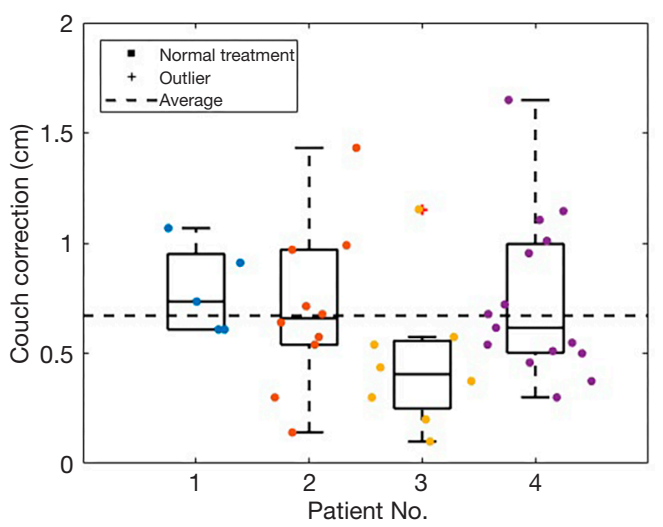

Figure 3 Couch corrections using daily kilovoltage cone-beam computed tomography. Mean three-dimensional vector couch correction from superficial marker alignment to daily kilovoltage cone-beam computed tomography for all fractions of all patients' setups on Halcyon ${ }^{\mathrm{TM}}$.

29 courses of palliative RT for MPM for pain, dyspnea, and dysphagia, among other symptoms, palliation was "complete or substantial" in $17 \%$ of courses, "moderately effective" in $21 \%$ of courses, and "inadequate" in $62 \%$ of courses (20). In another experience in the 1980s, in which
21 patients received 31 courses of palliative RT for MPM, predominantly for pain control, 17 (65\%) courses achieved at least partial palliation (21). While understanding the limitation that we had far fewer patients available for analysis than the previously published experiences, and a short follow-up interval (largely attributable to systemic disease-related deaths), our palliative outcomes compare well with such experiences.

Acute toxicity from palliative RT on Halcyon ${ }^{\mathrm{TM}}$ for MPM was relatively mild, with no (0\%) Grade 3 or higher toxicities, and two Grade 2 toxicities (fatigue, 25\%, $\mathrm{n}=1$, and cough, $25 \%, \mathrm{n}=1$ ), even with $75 \%$ of patients receiving concurrent systemic therapies, and $50 \%$ of patients having received prior thoracic $\mathrm{RT}$, which is a germane consideration in a frail (median ECOG 2), elderly, metastatic population in whom a key goal is to improve quality of life. While it is difficult to definitively show causation between a single treatment modality and the toxicities observed, it is likely that RT was at least partially contributory. Fatigue is a common side effect experienced during most RT courses, and most systemic therapy courses, with 3 of our patients receiving some form of systemic therapy concurrently with RT. Dyspnea and cough are symptoms experienced at baseline by many patients with 
Table 3 Beam-on, treatment, and approximate total treatment room times for each patient course

\begin{tabular}{lccc}
\hline Patient & Beam-on time $(\mathrm{min})^{\dagger}$ & Treatment time & Approximate total treatment room time \\
\hline 1 & 1.9 & 2.2 & 10.2 \\
2 & 1.6 & 1.8 & 9.8 \\
3 & 1.4 & 1.7 & 9.7 \\
4 & 1.4 & 1.7 & 9.7 \\
Mean \pm SD & $1.6 \pm 0.2$ & $1.8 \pm 0.2$ & $9.8 \pm 0.2$ \\
\hline
\end{tabular}

MPM, but could be exacerbated by any local inflammation caused to the tracheobronchial tree and/or esophagus during RT. Further, nausea was observed in a patient who received mediastinal RT for lymphadenopathy compressing a length of the esophagus and gastroesophageal junction, and in a patient receiving concurrent systemic therapy, which may independently cause nausea. Previous published reports of toxicity for palliative RT for MPM are sparse. Our experience compares well with one such experience of predominantly-palliative RT for MPM using conventional RT techniques, which reported one (0\%) Grade 3 or higher toxicity (non-fatal esophagitis), and 7-19\% rates of Grade 1-2 malaise, dyspnea, esophagitis, upper gastrointestinal toxicity, or dermatitis (19).

Dosimetrically, MPM RT plans on Halcyon ${ }^{\text {TM }}$ met all pre-specified target and OAR constraints, with the exception of one maximum esophageal dose, by $0.1 \mathrm{~Gy}$, in a patient who received prior thoracic RT and whose PTV was in the proximity of the esophagus. This was allowed at the discretion of the treating physician to maximize target coverage while meeting the cumulative spinal cord constraint.

Daily CBCT IGRT for MPM RT on Halcyon ${ }^{\mathrm{TM}}$, even without an optical distance indicator or source-to-surface distance confirmation, resulted in a mean $3 \mathrm{D}$ vector couch correction of $0.67 \pm 0.15 \mathrm{~cm}$, which compares well with previously published experiences of $3 \mathrm{D}$ vector couch corrections using daily MV or $\mathrm{kV}$ CBCT on Halcyon ${ }^{\mathrm{TM}}$ for patients with breast cancer $(0.77 \pm 0.05 \mathrm{~cm})(17)$ and gynecologic cancers $(0.90 \pm 0.37 \mathrm{~cm})(18)$, and daily $\mathrm{MV}$ CBCT on a TomoTherapy ${ }^{\circledR}$ linac for patients with MPM $(1.94 \mathrm{~cm})(22)$. The vector couch correction from the TomoTherapy ${ }^{\circledR}$ study was obtained by extracting the X, Y, and $\mathrm{Z}$ axis mean MV CBCT couch correction values from figure 4 of their analysis, and then calculating the mean vector from those values. While it is difficult to directly compare the couch corrections in our case series to those of the breast and gynecologic analyses, given different setups, treatment complexities, and targets, and the TomoTherapy ${ }^{\circledR}$ study given the use of MV CBCT rather than $\mathrm{kV}$ CBCT, and that PTV volumes were substantially larger in that study (mean 2,475.6 $\mathrm{cm}^{3}$ ) than this study (median $285.8 \mathrm{~cm}^{3}$ ), these values do provide a framework within which to consider the current results.

The mean beam-on, treatment, and approximated inroom times for MPM RT on Halcyon ${ }^{\mathrm{TM}}$ were $1.6 \pm 0.4$, $1.8 \pm 0.2$, and $9.8 \pm 0.2 \mathrm{~min}$, respectively. Limited published benchmark data of treatment times for RT for MPM exist. Two prior publications report treatment delivery times of 5.0-5.4 min for VMAT for MPM, but direct comparison is limited in that the treatment volumes in those studies encompassed the entire hemithorax, a much larger treatment volume than in our study, which could independently lengthen treatment time $(7,23)$. The beamon and treatment times observed compare favorably with those of patients treated for breast cancer $(2.0 \pm 0.3$ and $4.4 \pm 0.4 \mathrm{~min}$, respectively) and gynecologic cancers $(2.9 \pm 0.4$ and 3.6 \pm 0.4 min, respectively) on Halcyon ${ }^{\mathrm{TM}}$, while again recognizing the limitation of the differences in disease sites, setup details, and treatment volumes $(17,18)$. In this context, our results show quick beam-on, treatment, and approximate total room times for palliative MPM RT on Halcyon $^{\mathrm{TM}}$, and provide benchmark data for future MPM and/or palliative RT time comparisons.

While it has been expected that treatments on Halcyon $^{\mathrm{TM}}$ would be quick given its design properties, our case series is one of the first published clinical experiences to actually provide such data. This experience demonstrates that as in the breast and gynecologic cancer experiences $(17,18)$, treatment can be delivered with short treatment 
times, which has the potential to increase throughput within a department. Specifically, in this experience using Halcyon $^{\mathrm{TM}}$ to deliver palliative RT for MPM, effective symptomatic palliation was achieved with minimal toxicity. Short treatment times allow patients to be on the treatment table, which can be uncomfortable, for less time, potentially improving the patient experience. The ability to provide palliation quickly and safely in a frail, elderly, metastatic population, where quality of life is a priority, is highly valuable for patients and satisfying for providers.

Our report has multiple limitations. The small sample size may limit the generalizability of results. While symptom palliation was effective, the follow-up interval was short, and it is possible that with extended follow-up in patients who may have longer-term survival than the studied patients, recurrences of disease and/or symptoms may occur. Another limitation is that while the beam-on and treatment times were drawn from the delivered RT plans, the approximate total room time was extrapolated based upon data from breast RT on Halcyon ${ }^{\mathrm{TM}}$, which contained patients with better baseline performance statuses than those in our report. As such, it may take patients receiving palliative RT for MPM more time to transfer on and off of the treatment table, and to undergo setup shifts following IGRT, which could result in longer in-room times than estimated.

Herein we report a case series representing the initial clinical experience treating patients with palliative RT for MPM on Halcyon ${ }^{\mathrm{TM}}$, and one of the first reports of the clinical use of Halcyon ${ }^{\text {TM }}$. Such treatment provided symptom palliation across multiple palliative scenarios, with minimal toxicity, acceptable dosimetry, and setup corrections and treatment times that compared favorably with other published experiences of MPM RT. Thus, palliative RT on Halcyon ${ }^{\mathrm{TM}}$ can provide patients with MPM quick and safe tumor-related symptom relief, even in a frail, elderly population.

\section{Acknowledgments}

Funding: None.

\section{Footnote}

Reporting Checklist: The authors have completed the STROBE reporting checklist. Available at http://dx.doi. org/10.21037/apm-20-385

Data Sharing Statement: Available at http://dx.doi. org/10.21037/apm-20-385

Peer Review File: Available at http://dx.doi.org/10.21037/ apm-20-385

Conflicts of Interest: All authors have completed the ICMJE uniform disclosure form (available at http://dx.doi. org/10.21037/apm-20-385). Dr. LD and Dr. JMM report grants and personal fees from Varian Medical Systems, outside the submitted work. The other authors have no conflicts of interest to declare.

Ethical Statement: The authors are accountable for all aspects of the work in ensuring that questions related to the accuracy or integrity of any part of the work are appropriately investigated and resolved. This study was conducted in accordance with the Declaration of Helsinki (as revised in 2013). The study was approved by the institutional review board of the Hospital of the University of Pennsylvania (Protocol No. 831888) and individual consent for this retrospective analysis was waived.

Open Access Statement: This is an Open Access article distributed in accordance with the Creative Commons Attribution-NonCommercial-NoDerivs 4.0 International License (CC BY-NC-ND 4.0), which permits the noncommercial replication and distribution of the article with the strict proviso that no changes or edits are made and the original work is properly cited (including links to both the formal publication through the relevant DOI and the license). See: https://creativecommons.org/licenses/by-nc-nd/4.0/.

\section{References}

1. Robinson BM. Malignant pleural mesothelioma: an epidemiological perspective. Ann Cardiothorac Surg 2012;1:491-6.

2. Ettinger DS, Wood DE, Chair V, et al. NCCN Guidelines Panel Disclosures NCCN Guidelines Version 1.2020 Malignant Pleural Mesothelioma. 2019. Available online: https://www.nccn.org/professionals/physician_gls/pdf/ mpm.pdf

3. Gomez DR, Rimner A, Simone CB, et al. The Use of Radiation Therapy for the Treatment of Malignant Pleural Mesothelioma: Expert Opinion from the National Cancer Institute Thoracic Malignancy Steering Committee, International Association for the Study of Lung Cancer, and Mesothelioma Applied Research Foundation. J Thorac 
Oncol 2019;14:1172-83.

4. de Perrot M, Wu L, Wu M, et al. Radiotherapy for the treatment of malignant pleural mesothelioma. Lancet Oncol 2017;18:e532-42.

5. Gupta V, Mychalczak B, Krug L, et al. Hemithoracic radiation therapy after pleurectomy/decortication for malignant pleural mesothelioma. Int J Radiat Oncol Biol Phys 2005;63:1045-52.

6. Rosenzweig KE. Malignant pleural mesothelioma: adjuvant therapy with radiation therapy. Ann Transl Med 2017;5:242.

7. Runxiao L, Yankun C, Lan W. A pilot study of volumetricmodulated arc therapy for malignant pleural mesothelioma. J Appl Clin Med Phys 2016;17:139-44.

8. Kimura T, Doi Y, Nakashima T, et al. Clinical experience of volumetric modulated arc therapy for malignant pleural mesothelioma after extrapleural pneumonectomy. J Radiat Res 2015;56:315-24.

9. Michiels S, Poels K, Crijns W, et al. Volumetric modulated arc therapy of head-and-neck cancer on a fast-rotating O-ring linac: Plan quality and delivery time comparison with a C-arm linac. Radiother Oncol 2018;128:479-84.

10. Cashmore J. The characterization of unflattened photon beams from a $6 \mathrm{MV}$ linear accelerator. Phys Med Biol 2008;53:1933-46.

11. Li T, Irmen P, Liu H, et al. Dosimetric Performance and Planning/Delivery Efficiency of a Dual-Layer Stacked and Staggered MLC on Treating Multiple Small Targets: A Planning Study Based on Single-Isocenter Multi-Target Stereotactic Radiosurgery (SRS) to Brain Metastases. Front Oncol 2019;9:7.

12. Cozzi L, Fogliata A, Thompson S, et al. Critical Appraisal of the Treatment Planning Performance of Volumetric Modulated Arc Therapy by Means of a Dual Layer Stacked Multileaf Collimator for Head and Neck, Breast, and Prostate. Technol Cancer Res Treat 2018;17:1533033818803882.

13. Huang Y, Du Y, Li C, et al. Pediatric cone beam CT on Varian Halcyon and TrueBeam radiotherapy systems: radiation dose and positioning accuracy evaluations. J Radiol Prot 2019;39:739-48.

14. Flores-Martinez E, Kim GY, Yashar CM, et al. Dosimetric study of the plan quality and dose to organs at risk on tangential breast treatments using the Halcyon linac. J Appl Clin Med Phys 2019;20:58-67.
15. O'Grady F, Barsky AR, Anamalayil S, et al. Increase in Superficial Dose in Whole-Breast Irradiation With Halcyon Straight-Through Linac Compared With Traditional C-arm Linac With Flattening Filter: In vivo Dosimetry and Planning Study. Adv Radiat Oncol 2019;5:120-6.

16. Petroccia HM, Malajovich I, Barsky AR, et al. Spine SBRT With Halcyon $^{\mathrm{TM}}$ : Plan Quality, Modulation Complexity, Delivery Accuracy, and Speed. Front Oncol 2019;9:319.

17. Barsky AR, O'Grady F, Kennedy C, et al. Initial Clinical Experience Treating Patients with Breast Cancer on a 6-MV Flattening-Filter-Free O-Ring Linear Accelerator. Adv Radiat Oncol 2019;4:571-8.

18. Barsky AR, Kim MM, Dreyfuss A, et al. Initial clinical experience treating patients with gynecologic cancers on a $6 \mathrm{MV}$ flattening filter free O-ring linear accelerator. Adv Radiat Oncol 2020. doi:10.1016/j.adro.2020.01.004.

19. de Graaf-Strukowska L, Van Der Zee J, Van Putten W, et al. Factors influencing the outcome of radiotherapy in malignant mesothelioma of the pleura - A singleinstitution experience with 189 patients. Int J Radiat Oncol Biol Phys 1999;43:511-6.

20. Gordon W, Antman KH, Greenberger JS, et al. Radiation therapy in the management of patients with mesothelioma. Int J Radiat Oncol Biol Phys 1982;8:19-25.

21. Ball DL, Cruickshank DG. The Treatment of Malignant Mesothelioma of the Pleura. Am J Clin Oncol 1990;13:4-9.

22. Qi XS, Yang L, Lee P, et al. Fast, Low-Dose MegavoltageTopogram Localization on TomoTherapy: Initial Clinical Experience With Mesothelioma Patients. Pract Radiat Oncol 2019;9:373-80.

23. Dumane VA, Rimner A, Yorke ED, et al. Volumetricmodulated arc therapy for malignant pleural mesothelioma after pleurectomy/decortication. Appl Rad Oncol 2016;5:28-37.

Cite this article as: Barsky AR, Kim MM, Maxwell R, Mendes A, Wright CM, Anstadt EJ, McNulty S, Dong L, Metz JM, Feigenberg SJ, Li T, Cengel KA. Initial clinical experience treating patients with palliative radiotherapy for malignant pleural mesothelioma on the Halcyon ${ }^{\mathrm{TM}}$ linear accelerator. Ann Palliat Med 2020;9(5):2903-2912. doi: 10.21037/apm-20-385 